\title{
Divisibility of integer polynomials and tilings of the integers
}

\author{
by \\ ANDRÁs Biró (Budapest)
}

1. Introduction. If $A, B \subseteq \mathbb{Z}$ and every integer can be written uniquely as $a+b$ with $a \in A$ and $b \in B$, then we write $A \oplus B=\mathbb{Z}$, and we say that $A \oplus B$ is a tiling of $\mathbb{Z}$ by $A$. If $A \oplus B=\mathbb{Z}$ and $A$ is finite, it follows from the pigeon-hole principle (see e.g. $[\mathrm{N}]$ ) that $B$ is periodic, i.e. $B+k=B$ with some positive integer $k$.

We mention here that the analogous statement for $\mathbb{Z}^{r}$ in place of $\mathbb{Z}$ is an open problem (Periodic Tiling Conjecture, see [L-W]). Partial results are proved in $[\mathrm{Sz}]$. However, we deal here only with the case of $\mathbb{Z}$. For references to related work, see $[\mathrm{K}],[\mathrm{Sz}]$ and $[\mathrm{T}]$.

Let $A \oplus B=\mathbb{Z}$. If the diameter of $A$ is $n$, and the least period of $B$ is $k$, the pigeon-hole principle gives that $k \leq 2^{n}$ (see [N]). This result was recently improved by I. Z. Ruzsa, who proved in $[\mathrm{R}]$ that $\log k \ll \sqrt{n \log n}$. A slightly weaker result was proved by M. Kolountzakis in $[\mathrm{K}]$. In the other direction, the best known result is that $k=o\left(n^{2}\right)$ is not true (see $\left.[\mathrm{K}]\right)$. We see that these upper and lower estimates are very far from each other.

In the present paper we improve the upper bound (see the Corollary below), i.e. Ruzsa's result. However, the problem whether a polynomial upper bound can be given for $k$ or not, remains open.

We remark here that the proofs for the upper bound by Ruzsa and by Kolountzakis are nearly identical, except for a minor detail which makes the slight difference in the result. However, since I used [R] while I was working on my proof, I will refer to Ruzsa's work below.

The new upper bound will be a corollary of the following theorem on the divisibility of integer polynomials.

2000 Mathematics Subject Classification: 11C08, 11P99.

Research partially supported by the Hungarian National Foundation for Scientific Research (OTKA) Grants No. T032236, T042750, T043623, T049693 and D34576. 
TheOrem. Let $\varepsilon>0$ be any fixed constant, and let $0 \not \equiv q(x) \in \mathbb{Z}[x]$ with leading coefficient 1 . Assume that there is a polynomial $Q \not \equiv 0$ such that every coefficient of $Q$ is 0 or 1 and

$$
q(x) \mid(1-x) Q(x) .
$$

Denote by $n$ the degree of the polynomial $(1-x) Q(x)$, and assume that $n \geq n_{0}$, where $n_{0}$ is a large enough number depending only on $\varepsilon$. If there is a positive integer $k$ such that $q(x) \mid x^{k}-1$, then for the smallest such $k$ we have

$$
\log k \leq n^{1 / 3+\varepsilon} \text {. }
$$

REMARK. What is actually used about the polynomial $(1-x) Q(x)$ is that the sum of the absolute values of its coefficients is small, and the order of its vanishing at 1 is also small. So it would be possible to formulate the theorem imposing such conditions, and it would be nice to use more about $Q(x)$ in order to improve the result.

Corollary. Let $\varepsilon>0$ be any fixed constant, and let $A, B \subseteq \mathbb{Z}$ be such that $A$ is a finite set and $A \oplus B=\mathbb{Z}$. Write $n$ for the diameter of $A$, i.e. $n:=\max _{a \in A} a-\min _{a \in A} a$, and assume that $n \geq n_{0}$, where $n_{0}$ is a large enough number depending only on $\varepsilon$. Then there is a positive integer $k$ such that the set $B$ is periodic with respect to $k$ and

$$
\log k \leq n^{1 / 3+\varepsilon} .
$$

Examining Ruzsa's proof in $[R]$, we can see that the following statement on polynomials was proved there implicitly, and this was the basis of the proof of the result on tilings:

Proposition (Ruzsa). Let $q(x) \in \mathbb{Z}[x]$ with $\operatorname{deg} q \leq n$. If there is a positive integer $k$ such that $q(x) \mid x^{k}-1$, then for the smallest such $k$ we have $\log k \ll \sqrt{n \log n}$.

This result is sharp, as one can see from the example ( $p$ runs over primes)

$$
q(x)=\prod_{\sqrt{n \log n} \leq p \leq 2 \sqrt{n \log n}}\left(1+x+\cdots+x^{p-1}\right) .
$$

So the meaning of our Theorem is that if we assume a new condition on $q(x)$ (not only the upper bound for its degree), then the upper bound for $k$ can be improved. Since the polynomial $q(x)$ for which Ruzsa applied the above statement also satisfies the additional condition of our Theorem, the Corollary follows simply by substituting our Theorem into Ruzsa's proof. But, for the sake of completeness, we give the proof of the Corollary (i.e. we reconstruct Ruzsa's argument) at the end of the paper (Section 4).

The proof of some lemmas are presented in Section 2. We remark that Lemma 4 is crucial for the improvement of the exponent $1 / 2$ of $n$. We give 
the proof of the Theorem in Section 3. The proof begins by an analysis of Ruzsa's proof of the above Proposition. Then the new idea, leading to the improvement, is to substitute certain roots of unity into our polynomial, producing in this way nonzero and not very large integers divisible by too many primes, which will be a contradiction.

We introduce some notations. If $d \geq 1$ is an integer, $U(d)$ denotes the set of primitive $d$ th roots of unity. The cardinality of this set is $\phi(d)$ (Euler's function). The cyclotomic polynomial of order $d$ is $\psi_{d}(x):=\prod_{\xi \in U(d)}(x-\xi)$. We will use many times the fact that $\psi_{d}(x)$ is an irreducible polynomial in $\mathbb{Z}[x]$, and also the following fact: if $P_{1}(x), P_{2}(x) \in \mathbb{Z}[x], P_{1}(x) \mid P_{2}(x)$ and $P_{1}$ has leading coefficient 1 , then $P_{2}(x) / P_{1}(x) \in \mathbb{Z}[x]$ (Gauss' lemma). For a polynomial $P$ we denote by $\|P\|$ the sum of the absolute values of its coefficients; for a positive integer $r$ we write $\omega(r)$ for the number of distinct prime divisors of $r$. As usual, log will denote the natural logarithm.

Acknowledgements. I am grateful to R. Tijdeman for drawing my attention to this problem.

2. Some lemmas. The first lemma is needed for Lemma 2. We remark that, as the referee pointed out, Lemma 1 could be easily improved, if we took into account the dependence on $d$, but this would not improve the final result.

Lemma 1. Let $P \not \equiv 0$ be a polynomial with complex coefficients and $\operatorname{deg} P=n$. Let $d$ be a positive integer, assume that $x^{d}-1 \mid P(x)$, and let $P^{\star}(x)=P(x) /\left(x^{d}-1\right)$. Then

$$
\left\|P^{\star}\right\| \leq n\|P\| .
$$

Proof. Let

$$
P(x)=a_{0}+a_{1} x+\cdots+a_{n} x^{n}, \quad P^{\star}(x)=b_{0}+b_{1} x+\cdots+b_{n-d} x^{n-d} .
$$

It is easy to see that $([y]$ denotes the integer part of a real $y)$

$$
-b_{j}=\sum_{t=0}^{[j / d]} a_{j-t d},
$$

so $\left|b_{j}\right| \leq\|P\|$ for $j=0,1, \ldots, n-d$. Since $d \geq 1$, the lemma follows.

Lemma 2. Let $\varepsilon>0$ be any fixed constant, and let $P \not \equiv 0$ be a polynomial with complex coefficients. Assume that $\operatorname{deg} P \leq n$ with an $n \geq n_{0}$, where $n_{0}$ is a large enough number depending only on $\varepsilon$. Let $m$ be a positive integer satisfying $\psi_{m}(x) \mid P(x)$, and let $P^{\star}(x)=P(x) / \psi_{m}(x)$. Then

$$
\left\|P^{\star}\right\| \leq e^{n^{\varepsilon}}\|P\| \text {. }
$$


Proof. It is known that ( $\mu$ denotes the Möbius function)

$$
\psi_{m}(x)=\prod_{d \mid m}\left(x^{d}-1\right)^{\mu(m / d)} .
$$

We can write it as $\psi_{m}(x)=\psi_{m}^{\star}(x) / \psi_{m}^{\star \star}(x)$, where

$$
\psi_{m}^{\star}(x)=\prod_{d \mid m, \mu(m / d)=1}\left(x^{d}-1\right), \quad \psi_{m}^{\star \star}(x)=\prod_{d \mid m, \mu(m / d)=-1}\left(x^{d}-1\right) .
$$

Then the polynomial $P^{\star}$ can be written as

$$
P^{\star}(x)=\frac{P(x) \psi_{m}^{\star \star}(x)}{\prod_{d \mid m, \mu(m / d)=1}\left(x^{d}-1\right)},
$$

so the denominator here divides the numerator. Denote by $\tau(r)$ the number of divisors of a positive integer $r$. It is clear that $\operatorname{deg} \psi_{m}^{\star \star} \leq m \tau(m)$ and $\left\|\psi_{m}^{\star \star}\right\| \leq 2^{\tau(m)}$, so applying Lemma 1 repeatedly we get

$$
\left\|P^{\star}\right\| \leq\left(\|P\| 2^{\tau(m)}\right)(n+m \tau(m))^{\tau(m)} .
$$

Since $\phi(m) \leq n$ follows from $\psi_{m}(x) \mid P(x)$, and since it is well known that

$$
\phi(r)>r^{1-\varepsilon}, \quad \tau(r)<r^{\varepsilon}
$$

for large enough $r$, the lemma is proved.

The following lemma is well known, but since its proof requires only a few lines, we present the proof.

Lemma 3. Let $\varepsilon>0$ be any fixed constant, let $K$ be a real number and assume that $K \geq K_{0}$, where $K_{0}$ is a large enough number depending only on $\varepsilon$. Set $C=10^{5} \log K$. Then

$$
\sum_{1 \leq r \leq K} C^{\omega(r)} \leq K^{1+\varepsilon}
$$

Proof. Let

$$
f(r)=\prod_{p \mid r, p>P} e^{\lambda},
$$

where $p$ runs over primes and we will choose the positive parameters $P$ and $\lambda$ later. Then we have

$$
C^{\omega(r)} \leq e^{\lambda \pi(P)}\left(C e^{-\lambda}\right)^{\omega(r)} f(r)
$$

for every $r \geq 1$, where $\pi(P)$ is the number of primes not exceeding $P$. Obviously $f(m n) \leq f(m) f(n)$ for every $m, n \geq 1$, and so

$$
\frac{1}{K} \sum_{1 \leq r \leq K} f(r) \leq \sum_{1 \leq r \leq K} \frac{f(r)}{r} \leq \prod_{p \leq K}\left(1-\frac{f(p)}{p}\right)^{-1} \leq e^{2 \sum_{p \leq K} f(p) / p}
$$


provided $f(p) / p \leq 1 / 2$ for all $p$. This condition is true if $e^{\lambda} \leq P / 2$. Hence, using also that for large enough $K$ we have

$$
\sum_{p \leq K} \frac{1}{p} \leq 2 \log \log K \quad \text { and } \quad \omega(r) \leq 2 \frac{\log K}{\log \log K} \quad \text { for } 1 \leq r \leq K,
$$

we get

$$
\sum_{1 \leq r \leq K} C^{\omega(r)} \leq K\left(C e^{-\lambda}\right)^{2 \frac{\log K}{\log \log K}} e^{\lambda P+4 e^{\lambda} \log \log K}
$$

for large $K$, if $e^{\lambda} \leq P / 2$ and $e^{\lambda} \leq C$. By the choice

$$
P=2 e^{\lambda}, \quad \lambda=\log \log K-2 \log \log \log K,
$$

say, the lemma follows.

Lemma 4. Let $P \not \equiv 0$ be a polynomial such that every coefficient of $P$ is 0 or 1 and $\operatorname{deg} P=n$. Assume that $n \geq n_{0}$, where $n_{0}$ is a large enough number. Let $d$ be a positive integer. Then $\psi_{d}(x)^{V} \mid P(x)$ implies that $V \leq$ $(100 \log n)^{\omega(d)}$.

Proof. Let $d=d_{1} p^{\alpha}$, where $p$ is a prime, $\left(p, d_{1}\right)=1$ and $\alpha \geq 1$. For every integer $U \geq 0$ we have $\left(P^{(U)}\right.$ denotes the $U$ th derivative of $P$ )

$$
\psi_{d}(x)^{V-U} \mid P^{(U)}(x), \quad\left\|P^{(U)}\right\| \leq n^{U}(n+1) \leq n^{U+2} .
$$

Then

$$
\left(\prod_{\xi \in U\left(d_{1}\right)} \psi_{d}(\xi)\right)^{V-U} \mid N:=\prod_{\xi \in U\left(d_{1}\right)} P^{(U)}(\xi) .
$$

We know that $N \in \mathbb{Z}$ and $|N| \leq\left(n^{U+2}\right)^{\phi\left(d_{1}\right)}$. On the other hand,

$$
\prod_{\xi \in U\left(d_{1}\right)} \psi_{d}(\xi)=\prod_{\xi_{1}, \xi_{2} \in U\left(d_{1}\right)} \prod_{\eta \in U\left(p^{\alpha}\right)}\left(\xi_{1}-\xi_{2} \eta\right) .
$$

The right-hand side here is divisible by $p^{\phi\left(d_{1}\right)}$. Indeed, we see this from the $\xi_{1}=\xi_{2}$ part, using the fact that

$$
\prod_{\eta \in U\left(p^{\alpha}\right)}(1-\eta)=\psi_{p^{\alpha}}(1)=p
$$

Hence,

$$
\left(p^{\phi\left(d_{1}\right)}\right)^{V-U} \mid N \quad \text { and } \quad|N| \leq\left(n^{U+2}\right)^{\phi\left(d_{1}\right)} .
$$

If $N \neq 0$, this implies

$$
2^{V-U} \leq p^{V-U} \leq e^{(U+2) \log n} .
$$

If $V \geq 100 \log n$ and $U \leq V / 100 \log n$, then this is clearly a contradiction for large $n$, so $N$ must be 0 . 
We proved so far: if $n \geq n_{0}$ and

$$
\psi_{d}(x)^{V} \mid P(x), \quad V \geq 100 \log n, \quad U \leq \frac{V}{100 \log n},
$$

then

$$
\psi_{d_{1}}(x)^{U+1} \mid P(x) .
$$

Let $d=p_{1}^{\alpha_{1}} \cdots p_{t}^{\alpha_{t}}$ be the prime decomposition of the integer $d$. We apply the above step $t$ times. We conclude that if $V>(100 \log n)^{t}$, then $P(1)=0$, which is impossible by the assumptions. The lemma is proved.

3. Proof of the Theorem. We will use Lemmas 2-4 of Section 2.

In the present section the positive constants $c_{1}, c_{2}, \ldots$ are either absolute or depend only on $\varepsilon$; if a constant depends on $\varepsilon$, we will indicate it. If

$$
q(x) \mid x^{k}-1
$$

for some $k$, then

$$
q(x)=\prod_{d \in \mathcal{D}} \psi_{d}(x)
$$

with some set $\mathcal{D}$. Define

$$
k=\operatorname{lcm}\{d: d \in \mathcal{D}\},
$$

where lcm stands for the least common multiple. We have to estimate this $k$. Observe the important relation

$$
\operatorname{deg} q=\sum_{d \in \mathcal{D}} \phi(d) \leq n
$$

Let

$$
n^{1 / 3}<M<\frac{1}{2} n^{1 / 2}, \quad L>2 n^{1 / 2} .
$$

We will fix these parameters later.

(a) If $p$ is a prime, $\alpha \geq 1, p^{\alpha} \| k$ and $p^{\alpha} \geq L$, then $p^{\alpha} \mid d$ for some $d \in \mathcal{D}$. A given $d \in \mathcal{D}$ cannot be divisible by two different such prime powers, because then we would have (see also (2))

$$
\phi(d) \geq \phi\left(p_{1}^{\alpha_{1}}\right) \phi\left(p_{2}^{\alpha_{2}}\right) \geq\left(\frac{1}{2} p_{1}^{\alpha_{1}}\right)\left(\frac{1}{2} p_{2}^{\alpha_{2}}\right) \geq \frac{L^{2}}{4}>n,
$$

which contradicts (1). Hence every such prime power divides a different $d \in \mathcal{D}$, and if $p^{\alpha} \mid d$, then $\phi(d) \geq \phi\left(p^{\alpha}\right)$. So

$$
\frac{1}{2} \sum_{p^{\alpha} \| k, p^{\alpha} \geq L} L \leq \frac{1}{2} \sum_{p^{\alpha} \| k, p^{\alpha} \geq L} p^{\alpha} \leq \sum_{p^{\alpha} \| k, p^{\alpha} \geq L} \phi\left(p^{\alpha}\right) \leq \sum_{d \in \mathcal{D}} \phi(d) \leq n .
$$


This implies that for every such $p^{\alpha}$ we have $p^{\alpha} \leq 2 n$, and the number of such prime powers is $\leq 2 n / L$; consequently,

$$
\prod_{p^{\alpha} \| k, p^{\alpha} \geq L} p^{\alpha} \leq(2 n)^{2 n / L} .
$$

(b) It is clear that

$$
\prod_{p^{\alpha} \| k, p^{\alpha} \leq M} p^{\alpha} \leq \prod_{p^{\alpha} \leq M} p^{\alpha} \leq e^{c_{1} M} .
$$

(c) It is also clear that

$$
\prod_{p^{\alpha} \| k, M<p^{\alpha}<L, \alpha \geq 2} p^{\alpha} \leq \prod_{p^{\alpha}<L, \alpha \geq 2} p^{\alpha} \leq e^{c_{2} \sqrt{L}} .
$$

(d) We are left with

$$
\prod_{p \| k, M<p<L} p .
$$

If $p_{1}, p_{2}, p_{3}$ are three different such primes, and $p_{i} \mid d \in D$ for $i=1,2,3$, then

$$
\phi(d) \geq\left(p_{1}-1\right)\left(p_{2}-1\right)\left(p_{3}-1\right) \geq M^{3}>n
$$

by (2), which contradicts (1). So every such $p$ divides a $d \in \mathcal{D}$, but a given $d \in \mathcal{D}$ is divisible by at most two such primes. If $d \in \mathcal{D}$ is divisible by two such primes, then $\phi(d) \geq M^{2}$, so the number of such numbers $d \in \mathcal{D}$ is at most $n / M^{2}$ by (1). Consequently, the number of primes $p \| k$ with $M<p<L$ for which there is another such prime $p^{\star}$ and a $d \in \mathcal{D}$ with $p, p^{\star} \mid d$, is at most $2 n / M^{2}$. It follows that

$$
\prod_{p \| k, M<p<L} p \leq L^{2 n / M^{2}} \prod_{p \in \mathcal{P}} p,
$$

where $\mathcal{P}$ is a set

$$
\mathcal{P} \subseteq\{p \| k: M<p<L\}
$$

such that each $d \in \mathcal{D}$ is divisible by at most one $p \in \mathcal{P}$.

Then for every $p \in \mathcal{P}$ there is a $d_{p} \in \mathcal{D}$ with $p \mid d_{p}$, and $d_{p_{1}} \neq d_{p_{2}}$ for $p_{1} \neq p_{2}$. So by $(1)$, we obtain

$$
\sum_{p \in \mathcal{P}} \phi\left(d_{p}\right) \leq n
$$

Since $p \| k$ for $p \in \mathcal{P}$, we have $\left(p, d_{p} / p\right)=1$. Consequently,

$$
\phi\left(d_{p}\right)=(p-1) \phi\left(d_{p} / p\right) \geq c_{3}(\varepsilon) M\left(d_{p} / p\right)^{1-\varepsilon} .
$$

Introduce a new parameter $K \geq K_{0}$, where $K_{0}$ is a large number depending only on $\varepsilon$. If $p \in \mathcal{P}$ and $d_{p} / p \geq K$, then $\phi\left(d_{p}\right) \geq c_{3}(\varepsilon) M K^{1-\varepsilon}$ by 
(9), so we deduce by (7) and (8) that

$$
\prod_{p \in \mathcal{P}} p \leq\left(\prod_{p \in \mathcal{P}^{\star}} p\right) L^{c_{4}(\varepsilon) n / M K^{1-\varepsilon}},
$$

where $\mathcal{P}^{\star} \subseteq \mathcal{P}$ is a subset of $\mathcal{P}$ such that $1 \leq d_{p} / p<K$ for every $p \in \mathcal{P}^{\star}$.

We divide $\mathcal{P}^{\star}$ into subsets,

$$
\mathcal{P}^{\star}=\bigcup_{1 \leq r<K} P_{r},
$$

where

$$
P_{r}=\left\{p \in \mathcal{P}^{\star}: d_{p}=r p\right\} .
$$

Let $1 \leq r<K$ be fixed. Assume that $V_{r} \geq 0$ is the largest integer with the property

$$
\psi_{r}(x)^{V_{r}} \mid Q(x)(1-x),
$$

and let

$$
Q^{\star}(x)=\frac{Q(x)(1-x)}{\psi_{r}(x)^{V_{r}}} .
$$

Then $Q^{\star}(\xi) \neq 0$ for $\xi \in U(r)$, and by Lemma 2 we have

$$
\left\|Q^{\star}\right\| \leq 2 n e^{V_{r} n^{\varepsilon}},
$$

so, with the definition

$$
\nu:=\prod_{\xi \in U(r)} Q^{\star}(\xi)
$$

we have

$$
\nu \in \mathbb{Z}, \quad \nu \neq 0, \quad|\nu| \leq\left\|Q^{\star}\right\|^{\phi(r)} .
$$

Since, using (12),

$$
\prod_{p \in \mathcal{P}_{r}} \psi_{p r}(x)|q(x), \quad q(x)| Q(x)(1-x),
$$

and

$$
\left(\psi_{r}(x), \prod_{p \in \mathcal{P}_{r}} \psi_{p r}(x)\right)=1
$$

it follows that

$$
\prod_{p \in \mathcal{P}_{r}} \psi_{p r}(x) \mid Q^{\star}(x)
$$

by (14). Consequently,

$$
\prod_{p \in \mathcal{P}_{r}}\left(\prod_{\xi \in U(r)} \psi_{p r}(\xi)\right) \mid \nu
$$


by $(16)$. We have $(p, r)=1$ for every $p \in \mathcal{P}_{r}$, since $p \| k$ for every such $p$ (see (12), (7) and remember that $\left.\mathcal{P}_{r} \subseteq \mathcal{P}\right)$. Hence, for every $p \in \mathcal{P}_{r}$ we have

$$
\prod_{\xi \in U(r)} \psi_{p r}(\xi)=\prod_{\xi_{1}, \xi_{2} \in U(r)} \prod_{\eta \in U(p)}\left(\xi_{1}-\xi_{2} \eta\right) .
$$

Here the right-hand side is divisible by $p^{\phi(r)}$. This can be seen from the $\xi_{1}=\xi_{2}$ part, since

$$
\prod_{\eta \in U(p)}(1-\eta)=\psi_{p}(1)=p
$$

So the left-hand side is also divisible by $p^{\phi(r)}$. This is true for every $p \in \mathcal{P}_{r}$, and so by (18) we obtain

$$
\prod_{p \in \mathcal{P}_{r}} p^{\phi(r)} \mid \nu
$$

Then, taking into account (17) and (15), we get

$$
\prod_{p \in \mathcal{P}_{r}} p \leq\left\|Q^{\star}\right\| \leq 2 n e^{V_{r} n^{\varepsilon}}
$$

Since $p \geq 2$ for every $p$, this implies that for large enough $n$,

$$
\left|\mathcal{P}_{r}\right| \leq c_{5}\left(V_{r}+1\right) n^{\varepsilon} \text {. }
$$

Summing up: using the relations (3)-(7), $\mathcal{P}^{\star} \subseteq \mathcal{P},(10),(11)$ and (19) we infer that for large $n$,

$$
\begin{aligned}
\log k \leq & \frac{2 n}{L} \log (2 n)+c_{1} M+c_{2} \sqrt{L} \\
& +c_{6}(\varepsilon)(\log L)(K n)^{\varepsilon}\left(\frac{n}{M^{2}}+\frac{n}{M K}+\sum_{1 \leq r<K}\left(V_{r}+1\right)\right) .
\end{aligned}
$$

The $L$-part is (nearly) optimized by taking $L=n^{2 / 3}$. Using Lemma 4 , we see that $V_{r}+1 \leq(200 \log n)^{\omega(r)}$ for large $n$ (see the definition of $V_{r}$ at $\left.(13)\right)$. So, assuming a weak estimate

$$
K \geq n^{1 / 100}
$$

and using Lemma 3, we finally have

$$
\log k \leq c_{7}(\varepsilon)(K n)^{2 \varepsilon}\left(n^{1 / 3}+M+\frac{n}{M^{2}}+\frac{n}{M K}+K\right) .
$$

This is nearly optimized in $K$ by $K=(n / M)^{1 / 2}$, and the remaining expression is nearly optimized in $M$ with $M=n^{1 / 3}$. So finally we fix the parameters in the following way:

$$
K=n^{1 / 3}, \quad M=2 n^{1 / 3}, \quad L=n^{2 / 3} .
$$


Since the conditions (2) and (20) are satisfied with these choices (and the earlier assumption $K \geq K_{0}$ follows from (20)), we have

$$
\log k \leq n^{1 / 3+10 \varepsilon},
$$

say, for large enough $n$, so (writing $10 \varepsilon$ in place of $\varepsilon$ in the statement of the Theorem) the proof is complete.

4. Proof of the Corollary. We may assume that

$$
\min _{a \in A} a=0, \quad \max _{a \in A} a=n .
$$

Let

$$
\begin{aligned}
B^{+} & =B \cap[0, \infty), \\
C & =\{n \in \mathbb{Z}: n \geq 0, n=a+b, a \in A, b \in B, b<0\} .
\end{aligned}
$$

Define

$$
f(x)=\sum_{a \in A} x^{a}, \quad g(x)=\sum_{b \in B^{+}} x^{b}, \quad h(x)=\sum_{c \in C} x^{c} .
$$

Here $f$ and $h$ are polynomials. It is a consequence of $A \oplus B=\mathbb{Z}$ that

$$
f(x) g(x)+h(x)=\frac{1}{1-x},
$$

and so

$$
g(x)=\frac{1-(1-x) h(x)}{(1-x) f(x)}=\frac{p(x)}{q(x)},
$$

where $p$ and $q$ are coprime polynomials in $\mathbb{Z}[x]$. In particular, $q$ has leading coefficient 1 and

$$
q(x) \mid(1-x) f(x) .
$$

We know (from $[\mathrm{N}]$ ) that $B$ is periodic, therefore there is a positive integer $k$ for which $g(x)\left(x^{k}-1\right)$ is a polynomial. Then, since $(p(x), q(x))=1$, we have

$$
q(x) \mid x^{k}-1 .
$$

Since every coefficient of the polynomial $f$ is 0 or 1 , the conditions of the Theorem are fulfilled. We deduce from $\operatorname{deg} f=n$ that there is a positive integer $k$ with

$$
q(x) \mid x^{k}-1, \quad \log k \leq n^{1 / 3+\varepsilon} .
$$

It remains to prove that this $k$ is a period of $B$. But it is obvious that $g(x)\left(x^{k}-1\right)$ is a polynomial, and since we know that $B$ has a period (not necessarily this $k$, but some positive integer), in view of the definition of $g(x)$ this implies that $k$ is a period of $B$. 


\section{References}

[K] M. Kolountzakis, Translational tilings of the integers with long periods, Electron. J. Combin. 10 (2003), \#R22.

$[\mathrm{L}-\mathrm{W}] \quad$ J. C. Lagarias and Y. Wang, Tiling the line with translates of one tile, Invent. Math. 124 (1996), 341-365.

[N] D. J. Newman, Tesselations of integers, J. Number Theory 9 (1977), 107-111.

[R] I. Z. Ruzsa, Appendix to [T].

[Sz] M. Szegedy, Algorithms to tile the infinite grid with finite clusters, preprint, 1998, www.cs.rutgers.edu/ szegedy/

[T] R. Tijdeman, Periodicity and almost periodicity, preprint, 2002, www.math.leidenuniv.nl/ ${ }^{\text {tijdeman/preprints.html }}$
A. Rényi Institute of Mathematics
Hungarian Academy of Sciences
Reáltanoda u. 13-15
1053 Budapest, Hungary
E-mail: biroand@renyi.hu

Received on 15.8.2003

and in revised form on 19.7.2004 